\title{
Vibrational spectra of distorted structure macro \& nano molecules: An algebraic approach
}

\author{
Srinivasa Rao Karumuri ${ }^{1^{*}}$, J. Vijayasekhar ${ }^{2}$, Velagapudi Uma Maheswara Rao ${ }^{3}$, \\ Ganganagunta Srinivas ${ }^{4}$, Aappikatla Hanumaiah ${ }^{5}$ \\ ${ }^{1}$ Department of Electronics \& Instrumentation, Lakireddy Bali Reddy College of Engineering, Mylavaram, India; \\ *Corresponding Author: srinivasakarumuri@gmail.com \\ ${ }^{2}$ Department of Mathematics, GITAM University, Hyderabad, India \\ ${ }^{3}$ Department of Applied Mathematics, Andhra University, Vishakhapatnam, India \\ ${ }^{4}$ Department of Physics, KL University, Guntur, India \\ ${ }^{5}$ Department of Sciences \& Humanities, Lara Vigyan Institute of Science \& Technology, Vadlamudi, India
}

Received 18 April 2012; revised 20 June 2012; accepted 10 July 2012

\begin{abstract}
Using the Lie algebraic method the vibrational frequencies of 97 resonances Raman lines $\left(A_{1 g}+\right.$ $\left.B_{1 g}+A_{2 g}+B_{2 g}\right)$ and 38 infrared bands $\left(E_{u}\right)$ of octaethylporphyrinato-Ni (II) and its mesodeuterated and ${ }^{15} \mathrm{~N}$-substituted derivates and Fullerenes $\mathrm{C}_{60}$ and $\mathrm{C}_{70}$ of 7 vibrational bands are calculated using $U(2)$ algebraic Hamiltonian with four fitting algebraic parameters. The results obtained by the algebraic technique have been compared with experimental data; and they show great accuracy.
\end{abstract}

Keywords: Lie Algebra; Vibrational Spectra; $\mathrm{Ni}$ (OEP); Ni (OEP)- $\mathrm{d}_{4} \& \mathrm{Ni}(\mathrm{OEP})-\mathrm{N}_{4}$; Fullerenes

\section{INTRODUCTION}

Nanoscience is an interdisciplinary field that seeks to bring about nature nanotechnology. Focusing on the nanoscale intersection of fields such as Physics, Biology, Engineering, Chemistry, Computer Sciences and more, Nanoscience is rapidly expanding [1]. A comprehensive treatment and understanding of spectroscopic features of nano-size molecules is by far one of the most challenging aspects of current studies in molecular spectroscopy. On one side, experimental techniques are producing a rapidly increasing amount of data and clear evidence for intriguing mechanisms characterizing several aspects of molecular dynamics in nano-bio molecules [2]. On the other side, theoretical approaches are heavily pushed towards their intrinsic limits; in the attempt to provide reliable answers to hitherto unresolved questions concerning very complex situations of nanobio molecules. The appearance of new experimental techniques to produce higher vibrational excitations in nano-bio polyatomic molecule requires reliable theoretical methods for their interpretation. Two approaches have mostly been used so far in an analysis of experimental data: 1) the familiar Dunham like expansion of energy levels in terms of rotations-vibrations quantum numbers and 2) the solution of Schrodinger equation with potentials obtained either by appropriately modifying ab-initio calculations or by more phenomenological methods. In this article, we begin a systematic analysis of vibrational spectra of bio-nano molecules in terms of novel approach; 3) Vibron model [3-6].

Recently Lie algebraic model introduction [7-18] could proved itself to be a successful model in the study of vibrational spectra of small, medium size and polyatomic molecules $[19,20]$. The algebraic model is fully based on the dynamical symmetry and through the language of Lie algebra. For the triatomic, tetratomic, Tetrahedral and poly-atomic Bio-molecules (i.e. metalloporphyrins, Ni (OEP), Ni (TTP), Ni Porphyrin) we studied earlier [21-25] using algebraic model. Using the algebraic model in this study we have calculated the vibrational frequencies of octaethylporphyrinato-Ni(II) and its meso-deurated and $\mathrm{N}$ substituted derivatives for 97 vibrational bands each using $U(2)$ algebraic model Hamiltonian. In our study we used four fitting parameters which provide better comparisons between the experimental and theoretical calculations throughout the study.

In this paper, we have considered only the In-Plane Vibrations of Nickel Octaethylporphyrin and its meso substituent and ${ }^{15} \mathrm{~N}$ derivatives for 97 vibrational bands and fullerenes $\mathrm{C}_{60}$ and $\mathrm{C}_{70}$ for 7 vibrational bands (both stretching and bending) are calculated by using $U(2)$ algebraic mode Hamiltonian. 


\section{ALGEBRAIC FRAMEWORK}

A complete description of the theoretical foundations needed to formulate the algebraic model for a vibrating molecule. We apply the one-dimensional algebraic model, consisting of a formal replacement of the interatomic, bond coordinates with unitary algebras. To say it in different words, the second-quantization picture suited to describe anharmonic vibrational modes, is specialized through an extended use of Lie group theory and dynamical symmetries. By means of this formalism, one can attain algebraic expressions for eigenvalues and eigenvectors of even complex Hamiltonian operators, including intermode coupling terms as well expectation values of any operator of interest (such as electric dipole and quadrupole interactions). Algebraic model are not $a b$-initio methods, as the Hamiltonian operator depends on a certain number of a priori undetermined parameters. As a consequence, algebraic techniques can be more convincingly compared with semi-empirical approaches making use of expansions over power and products of vibrational quantum numbers, such as a Dunham-like series. However, two noticeable advantages of algebraic expansions over conventional ones are that 1) algebraic modes lead to a (local) Hamiltonian formulation of the physical problem at issue (thus permitting a direct calculation of eigenvectors in this same local basis) and 2) algebraic expansions are intrinsically anharmonic at their zero-order approximation. This fact allows one to reduce drastically the number of arbitrary parameters in comparison to harmonic series, especially when facing medium- or large-size molecules. It should be however also noticed that, as a possible drawback of purely local Hamiltonian formulations (either algebraic or not) compared with traditional perturbative approaches, the actual eigenvectors of the physical system. Yet, for very local situations, the aforementioned disadvantage is not a serious one. A further point of import here is found in the ease of accounting for proper symmetry adaptation of vibrational wave functions. This can be of great help in the systematic study of highly excited overtones of not-so-small molecules, such as the present one. Last but not least, the local mode picture of a molecule is enhanced from the very beginning within the algebraic framework. This is an aspect perfectly lined up with the current tendencies of privileging local over normal mode pictures in the description of most topical situations.

We address here the explicit problem of the construction of the vibrational Hamiltonian operator for the polyatomic molecule. According to the general algebraic description for one-dimensional degrees of freedom, a dynamically-symmetric Hamiltonian operator for $\mathrm{n}$ interacting (not necessarily equivalent) oscillators cab written as

$$
H=E_{0}+A_{i} C_{i}+A_{i j} C_{i j}+\lambda_{i j} M_{i j} .
$$

In this expresssion, one finds three different classes of effective contributions. The first one, $\sum_{i=1}^{n} A_{i} C_{i}$ is devoted to the description of $n$ independent, anharmonic sequences of vibrational levels (associted wih $n$ independent, local oscillator) in terms of the operators $C_{i}$. The second one, $\sum_{i=1}^{n} A_{i j} C_{i j}$ leads to cross-anharmonicities between pairs of distinct local oscillators in terms of the operators $C_{i j}$. The third one, $\sum_{i=1}^{n} \lambda_{i j} M_{i j}$, describes anharmonic, non-diagonal interactions involving pairs of local oscillators in terms of the operators $M_{i j}$. The $C_{i}, C_{i j}$ operators are invariant (Casimir) operators of certain Lie algebras, whilst the $M_{i j}$ are invariant (Majorana) operators associated with coupling schemes involving algebras naturally arising from a systematic study of the algebraic formulation of the one-dimensional model for $n$ interacting oscillators. We work in the local (uuncoupled oscillaators) vibrational basis written as

$$
|v\rangle \equiv\left|v_{1} v_{2} v_{3} \ldots \ldots v_{n}\right\rangle \text {. }
$$

In which the aforementioned operators have the following matrix elements

$$
\begin{aligned}
\left\langle v\left|C_{i}\right| v\right\rangle= & -4 v_{i}\left(N_{i}-v_{i}\right) \\
\left\langle v\left|C_{i j}\right| v\right\rangle= & -4\left(v_{i}+v_{i}\right)\left(N_{i}+N_{j}-v_{i}-v_{j}\right) \\
\left\langle v^{!}\left|M_{i j}\right| v\right\rangle= & \left(v_{i} N_{i}+v_{j} N_{j}-2 v_{i} v_{j}\right) \delta_{v_{i}^{\prime} v_{i}} \delta_{v_{j}^{\prime} v_{j}} \\
\left\langle v^{!}\left|M_{i j}\right| v\right\rangle= & -\left[\left(v_{i}+1\right)\left(N_{i}-v_{i}\right) v_{j}\left(N_{j}-v_{j}+1\right)\right]^{1 / 2} \\
& \times \delta_{v_{i}^{\prime}-1 v_{i}} \delta_{v_{j}^{\prime}+1 v_{j}} \\
\left\langle v^{!}\left|M_{i j}\right| v\right\rangle= & -\left[\left(v_{j}+1\right)\left(N_{j}-v_{j}\right) v_{i}\left(N_{i}-v_{i}+1\right)\right]^{1 / 2} \\
& \times \delta_{v_{i}^{\prime}+1 v_{i}} \delta_{v_{j}^{\prime}-1 v_{j}} .
\end{aligned}
$$

We note, in particular, that the expressions above depend on the numbers $N_{i}$ (Vibron numbers). Such numbers have to be seen as predetermined parameters of welldefined physical meaning, as they relate to the intrinsic anharmonicity of a single, uncoupled oscillator through the simple relation. We report in Table the values of the Vibron numbers used in the present study.

The general Hamiltonian operator 1) can be adapted to describe he internal, vibrational degrees of freedom of any polyatomic molecule in two distinct steps. First, we associate three mutually perpendicular one-dimensional anharmonic oscillators to each atom. This procedure eventually leads to a redundant picture of the whole molecule, as it will include spurious (i.e. translational/rotational) degrees of freedom. It is however possible to remove easily such spurious modes through a technique de- 
scribed elsewhere [19-21]. One is thus left with a Hamiltonian operator dealing only with true vibrations. Such modes are given in terms of coupled oscillators in the local basis; 2) The coupling is induced by the Majorana operators. A sensible use of these operators is such that the correct symmetries of vibrational wave functions are properly taken into account. As a second step, the algebraic parameters $A_{i}, A_{i j}, \lambda_{i j}$ of Eq.1 need to be calibrated to reproduce the observed spectrum.

The algebraic theory of polyatomic molecules consists in the separate quantization of rotations and vibrations in terms of vector coordinates $r_{1}, r_{2}, r_{3}, \cdots$. quantized through the algebra

$$
G \equiv U_{1}(2) \otimes U_{2}(2) \otimes U_{3}(2) \otimes \cdots
$$

For the stretching vibrations of polyatomic molecules correspond to the quantization of anharmonic Morse oscillators, with classical Hamiltonian

$$
H\left(p_{s}, s\right)=p_{s}^{2} / 2 \mu+D[1-\exp (-\beta s)]^{2}
$$

For each oscillator $i$, states are characterized by representations of

$$
\left|\begin{array}{cc}
U_{i}(2) & \supset \\
\downarrow & O_{i}(2) \\
N_{i} & m_{i}
\end{array}\right|
$$

with $m_{i}=N_{i}, N_{i}-2, \cdots, 1$ or 0 ( $N_{i}$-odd or even). The Morse Hamiltonian (3) can be written, in the algebraic approach, simply as

$$
H_{i}=\varepsilon_{0 i}+A_{i} C_{i},
$$

where $C_{i}$ is the invariant operator of $O_{i}(2)$, with eigen values

$$
\varepsilon_{i}=\varepsilon_{0 i}+A_{i}\left(m_{i}^{2}-N_{i}^{2}\right) .
$$

Introducing the vibrational quantum number $v_{i}=\left(N_{i}-m_{i}\right) / 2,[20]$ one has

$$
\varepsilon_{i}=\varepsilon_{0 i}-4 A_{i}\left(N_{i} v_{i}-v_{i}^{2}\right)
$$

For non-interacting oscillators the total Hamiltonian is

$$
H=\sum_{i} H_{i}
$$

with eigen-values

$$
E=\sum_{i} \varepsilon_{i}=E_{0}-\sum_{i} 4 A_{i}\left(N_{i} v_{i}-v_{i}^{2}\right)
$$

\subsection{Hamiltonian for Stretching Vibrations}

The interaction potential can be written as

$$
V\left(s_{i}, s_{j}\right)=k_{i j}^{\prime}\left[1-\exp \left(-\alpha_{i} s_{i}\right)\right]\left[1-\exp \left(-\alpha_{j} s_{j}\right)\right],
$$

which reduces to the usual harmonic force field when the displacements are small

$$
V\left(s_{i}, s_{j}\right) \approx k_{i j} s_{i} s_{j} .
$$

Interaction of the type $\mathbf{E q . 8}$ can be taken into account in the algebraic approach by introducing two terms. One of these terms is the Casimir operator, $C_{i j}$, of the combined $O_{i}(2) \otimes O_{j}(2)$ algebra. The matrix elements of this operator in the basis Eq.2 are given by

$$
\begin{aligned}
& \left\langle N_{i}, v_{i}, N_{j}, v_{j}\left|C_{i j}\right| N_{i}, v_{i}, N_{j}, v_{j}\right\rangle ? \\
= & 4\left[\left(v_{i}+v_{j}\right)^{2}-\left(v_{i}+v_{j}\right)\left(N_{i}+N_{j}\right)\right] .
\end{aligned}
$$

The operator $C_{i j}$ is diagonal and the vibrational quantum numbers $v_{i}$ have been used instead of $m_{i}$. In practical calculations, it is sometime convenient to substract from $C_{i j}$ a contribution that can be absorbed in the Casimir operators of the individual modes $i$ and $j$, thus considering an operator $C_{i j}^{\prime}$ whose matrix elements are

$$
\begin{aligned}
& \left\langle N_{i}, v_{i} ; N_{j}, v_{j}\left|C_{i j}\right| N_{i}, v_{i} ; N_{j}, v_{j}\right\rangle \\
= & 4\left[\left(v_{i}+v_{j}\right)^{2}-\left(v_{i}+v_{j}\right)\left(N_{i}+N_{j}\right)\right] \\
+ & {\left[\left(N_{i}+N_{j}\right) / N_{i}\right] 4\left(N_{i} v_{i}-v_{i}^{2}\right) } \\
+ & {\left[\left(N_{i}+N_{j}\right) / N_{i}\right] 4\left(N_{j} v_{j}-v_{j}^{2}\right) . }
\end{aligned}
$$

The second term is the Majorana operator, $M_{i j}$. This operator has both diagonal and off-diagonal matrix elements

$$
\begin{aligned}
& \left\langle N_{i}, v_{i} ; N_{j}, v_{j}\left|M_{i j}\right| N_{i}, v_{i} ; N_{j}, v_{j}\right\rangle \\
= & \left(N_{i} v_{j}+N_{j} v_{i}-2 v_{i} v_{j}\right) \\
& \left\langle N_{i}, v_{i}+1 ; N_{j}, v_{j}-1\left|M_{i j}\right| N_{i}, v_{i} ; N_{j}, v_{j}\right\rangle \\
= & -\left[v_{j}\left(v_{i}+1\right)\left(N_{i}-v_{i}\right)\left(N_{j}-v_{j}+1\right)\right]^{1 / 2} \\
& \left\langle N_{i}, v_{i}-1 ; N_{j}, v_{j}+1\left|M_{i j}\right| N_{i}, v_{i} ; N_{j}, v_{j}\right\rangle \\
= & -\left[v_{i}\left(v_{j}+1\right)\left(N_{j}-v_{j}\right)\left(N_{j}-v_{i}+1\right)\right]^{1 / 2} .
\end{aligned}
$$

The Majorana operators $M_{i j}$ annihilâtes one quantum of vibration in bond $i$ and create one in bond $j$, or vice versa.

\subsection{Symmetry-Adapted Operators}

In polyatomic molecules, the geometric point group symmetry of the molecule plays an important role. States must transform according to representations of the point symmetry group. In the absence of the Majorana operators $M_{i j}$, states are degenerate. The introduction of the Majorana operators has two effects: 1) It splits the de- 
generacies of figure and 2) in addition it generates states with the appropriate transformation properties under the point group. In order to achieve this result the $\lambda_{i j}$ must be chosen in an appropriate way that reflects the geometric symmetry of the molecule. The total Majorana operator

$$
S=\sum_{i<j}^{n} M_{i j}
$$

is divided into subsets reflecting the symmetry of the molecule

$$
S=S^{\prime}+S^{\prime}+\cdots
$$

The operators $S=S^{\prime}+S^{\prime}+\cdots$ are the symmetryadapted operators. The construction of the symmetryadapted operators of any molecule will become clear in the following sections where the cases of Metalloporphyrins $\left(\mathrm{D}_{4 \mathrm{~h}}\right)$ will be discussed.

\subsection{Hamiltonian for Bending Vibrations}

We emphasize once more that the quantization scheme of bending vibrations in $U(2)$ is rather different from $U(4)$ and implies a complete separation between rotations and vibrations. If this separation applies, one can quantize each bending oscillator $i$ by means of an algebra $U_{i}(2)$ as in Eq.4. The Poschl-Teller Hamiltonian

$$
H\left(p_{s}, s\right)=|2 \mu-D| \cosh ^{2}(\alpha s)
$$

where we have absorbed the $\lambda(\lambda-1)$ part into $D$, can be written, in the algebraic approach, as

$$
H_{i}=\varepsilon_{0 i}+A_{i} C_{i},
$$

This Hamiltonian is identical to that of stretching vibration (Eq.5). The only difference is that the coefficients $A_{i}$ in front of $C_{i}$ are related to the parameters of the potential, $D$ and $\alpha$, in a way that is different for Morse and Poschl-Teller potentials. The energy eigen-values of uncoupled Poschl-Teller oscillators are, however, still given by

$$
E=\sum_{i} \varepsilon_{i}=E_{0}-\sum_{i} 4 A_{i}\left(N_{i} v_{i}-v_{i}^{2}\right) .
$$

One can then proceed to couple the oscillators as done previously and repeat the same treatment.

\subsection{The Metalloporphyrins Molecule}

The construction of the symmetry-adapted operators and of the Hamiltonian operator of polyatomic molecules will be illustrated using the example of Metalloporphyrins. In order to do the construction, draw a figure corresponding to the geometric structure of the molecule (Figure 1). Number of degree of freedom we wish to describe.

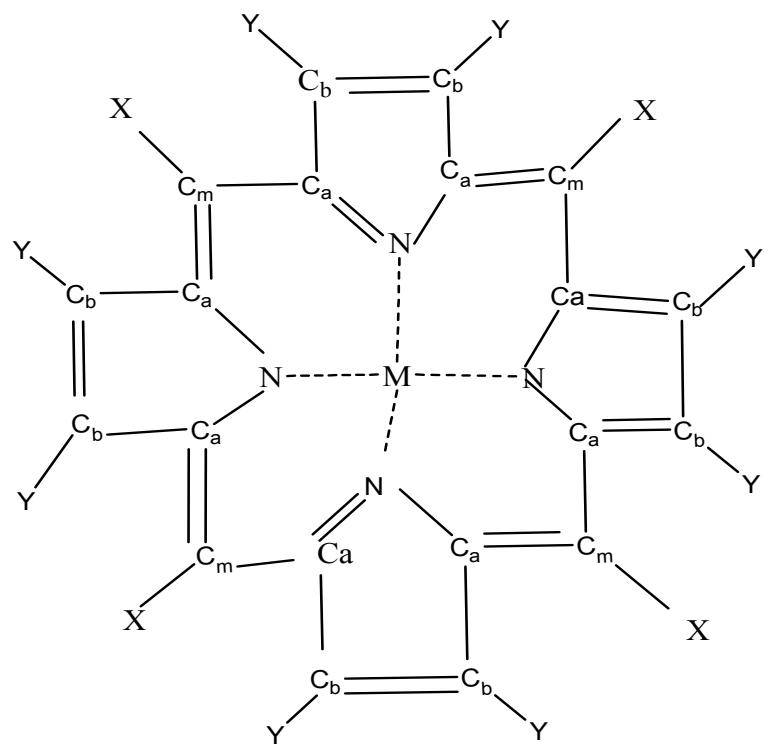

Figure 1. Structure of Metalloporphyrins.

By inspection of the figure, one can see that two types of interactions in Metalloporphyrins:

1) First-neighbor couplings (Adjacent interactions)

2) Second-neighbor couplings (Opposite interactions)

The symmetry-adapted operators of Metalloporphyrins with symmetry $\mathrm{D}_{4 \mathrm{~h}}$ are those corresponding to these two couplings, that is,

$$
S^{\prime}=\sum_{i<j}^{n} c_{i j}^{\prime} M_{i j}, S^{\prime \prime}=\sum_{i<j}^{n} c_{i j}^{\prime \prime} M_{i j},
$$

with

$$
\begin{aligned}
& c_{12}^{\prime}=c_{23}^{\prime}=c_{34}^{\prime}=c_{45}^{\prime}=\cdots=1 \\
& c_{13}^{\prime}=c_{24}^{\prime}=c_{35}^{\prime}=c_{46}^{\prime}=\cdots=0 \\
& c_{12}^{\prime \prime}=c_{23}^{\prime \prime}=c_{34}^{\prime \prime}=c_{45}^{\prime \prime}=\cdots=0 \\
& c_{13}^{\prime \prime}=c_{24}^{\prime \prime}=c_{35}^{\prime \prime}=c_{46}^{\prime \prime}=\cdots=1
\end{aligned}
$$

The total Majorana operator $\mathrm{S}$ is the sum

$$
S=S^{1}+S^{11}
$$

Diagonalization of $S$ produces states that carry representations of $S$, the group of permutations of objects, while Diagonalization of the other operators produces states that transform according to the representations $A_{1 g}$, $\mathrm{A}_{2 \mathrm{~g}}, \mathrm{~B}_{1 \mathrm{~g}}, \mathrm{~B}_{2 \mathrm{~g}}$ and $\mathrm{E}_{1 \mathrm{u}}$ of $\mathrm{D}_{4 \mathrm{~h}}$.

\subsection{Local to Normal Transition: The Locality Parameter $(\xi)$}

The local-to-normal transition is governed by the dimensionless locality parameter $(\xi)$. The local-to-normal transition can be studied $[19,20]$ for polyatomic molecules, for which the Hamiltonian is

$$
H=H^{\text {local }}+\lambda_{12} M_{12}=A_{i} C_{i}+A_{i j} C_{i j}+\lambda_{i j} M_{i j}
$$


For these molecules, the locality parameters are

$$
\xi_{i}=(2 / \Pi) \tan ^{-1}\left[8 \lambda_{i j} /\left(A_{i}+A_{i j}\right)\right], i, j=1,2,3 .
$$

Corresponding to the two bonds. $A$ global locality parameter for XYZ molecules can be defined as the geometric mean [20]

$$
\xi=\left(\left|\xi_{1} \xi_{2}\right|\right)^{1 / 2} .
$$

Locality parameters of this metalloporphyrins is given in the results and discussions With this definition, due to Child and Halonen [21], local-mode molecules are near to the $\xi=0$ limit, normal mode molecules have $\xi \rightarrow 1$.

\section{RESULTS AND DISCUSSIONS}

The number $N$ [total number of bosons, label of the ire-ducible representation of $U(4)]$ is related to the total number of bound states supported by the potential well. Equivalently it can be put in a one-to-one corresponddence with the anharmonicity parameters $x_{e}$ by means of

$$
x_{e}=\frac{1}{N+2} \text {. }
$$

We can rewrite the Eq.22 as

$$
N_{i}=\frac{\omega_{e}}{\omega_{e} x_{e}}-1(i=1,2 \cdots) .
$$

Now, for a blood cell molecule, we can have the values of $\omega_{e}$ and $\omega_{e} x_{e}$ for the distinct bonds (say CH, CC, $\mathrm{CD}, \mathrm{CN}$ etc.) from the study of K. Nakamoto [22] and that of K.P. Huber and G. Herzberg [23]. Using the values of $\omega e$ and wexe for the bond $\mathrm{CH} / \mathrm{CC}$ we can have the initial guess for the value of the vibron number $N$.

Depending on the specific molecular structure $\mathrm{N}_{\mathrm{i}}$ can vary between $\pm 20 \%$ of the original value. The vibron number $\mathrm{N}$ between the diatomic molecules $\mathrm{C}-\mathrm{H}$ and $\mathrm{C}-\mathrm{C}$ are 44 and 140 respectively. Since the bonds are equivalent, the value of $N$ is kept fixed. This is equivalent to change the single-bond anharmonicity according to the specific molecular environment, in which it can be slightly different.

Again the energy expression for the single-oscillator in fundamental mode is

$$
E(v=1)=-4 A(N-1)
$$

In the present case we have three and six different energies corresponding to symmetric and antisymmetric combinations of the different local mode.

$$
A=E / 4(1-N)
$$

where $E=$ Average energy, The initial guess for $\lambda$ can be obtained by

$$
\lambda=\left|E_{1}-E_{2}\right| / 2 N
$$

A numerical fitting procedure is adopted to adjust the parameters $A$ and $\lambda$ starting from the values above and $A^{\prime}$ whose initial guess can be zero.

The complete Calculation data in stretching and bending modes of different Bio \& Nano molecules are presented in Tables 1-5 and the corresponding algebraic parameters are presented in Tables $\mathbf{6}$ and $\mathbf{7 .}$

\section{CONCLUSIONS}

We have presented here a vibrational analysis of the stretching/bending modes of Bio molecules (i.e. Nickel Porphyrins) and Nano molecules (Fullerenes $\mathrm{C}_{60}, \mathrm{C}_{70}$ ) in terms of one-dimensional Vibron model i.e. $\mathrm{U}(2)$ algebraic model.

From the view of group theory, the molecule of $\mathrm{Ni}(\mathrm{OEP}), \mathrm{Ni}(\mathrm{OEP})-\mathrm{d}_{4} \& \mathrm{Ni}(\mathrm{OEP})-{ }^{15} \mathrm{~N}_{4}$ takes a square planar structure with the $\mathrm{D}_{4 \mathrm{~h}}$ symmetry point group. Molecular vibrations of metalloporphyrins are classified into the in-plane and out of plane modes. For Octaethyl dimmers of $\mathrm{D}_{4 \mathrm{~h}}$ structure assuming the peripheral ethyl group is point mass the in-plane vibrations of Octaethyl dimmers are factorized into 35 gerade and 18 ungerade. Out of planes are factorized into 8 gerade and 18 ungerade modes. The $\mathrm{A}_{2 \mathrm{u}}$ and $\mathrm{E}_{\mathrm{u}}$ modes are IR active where the $A_{1 g}, B_{1 g}, A_{2 g}, B_{2 g} \& E_{g}$ modes are Raman active in an ordinary sense. The Nano-molecules $\mathrm{C}_{60}$ and $\mathrm{C}_{70}$ are $\mathrm{I}_{\mathrm{h}}$ and $\mathrm{D}_{5 \mathrm{~h}}$ point group symmetry respectively.

In this study the resonance Raman spectra of Ni(OEP), $\mathrm{Ni}(\mathrm{OEP})-\mathrm{d}_{4}$ and $\mathrm{Ni}(\mathrm{OEP}){ }^{15} \mathrm{~N}_{4}$ for 97 vibrational bands, we obtain the RMS deviation i.e. $\Delta$ (r.m.s) $=40.92 \mathrm{~cm}^{-1}$, $33.03 \mathrm{~cm}^{-1}, 4.04 \mathrm{~cm}^{-1}$ and the locality parameters are $\xi_{1}$ $=0.0765, \xi_{2}=0.0468, \xi_{3}=0.0685$ respectively.

In this study the vibrational frequencies of Nano molecules $\mathrm{C}_{60}$ and $\mathrm{C}_{70}$ for 7 vibrational bands, we obtain the RMS deviation i.e. $\Delta$ (r.m.s) $=6.439 \mathrm{~cm}^{-1}, 3.2029$ $\mathrm{cm}^{-1}$, and the locality parameters are $\xi_{1}=0.0384, \xi_{2}=$ $0.0493, \xi_{3}=0.0590$ respectively.

Using improved set of algebraic parameters, the RMS deviation we reported in this study for Bio and Nano molecule is lying near about the experimental accuracy. Using only four algebraic parameters, the RMS deviation we reported in this study for Bio-Nano molecule are better fit.

The above two points confirm that in four parameters fit, the set of algebraic parameters we reported in this study of local to normal transition provide the best fit to the spectra of Bio-Nano molecules.

We hope that this work will be stimulate further research in analysis of vibrational spectra of other Nano molecules like fullerenes and protein molecules where the algebraic approach has not been applied so far. 
Table 1. Comparison between the experimental and Calculated frequencies of the resonance Raman active fundamental modes of $\mathrm{Ni}(\mathrm{OEP})\left(\mathrm{cm}^{-1}\right)$.

\begin{tabular}{|c|c|c|c|c|c|}
\hline Symmetry & Mode & Description & $\operatorname{Exp}^{\mathrm{a}}$ & Cal & $\Delta($ Exp-Calc $)$ \\
\hline \multirow{9}{*}{$\mathrm{A}_{1 \mathrm{~g}}$} & $v 1$ & $v(\mathrm{Cm}-\mathrm{H})$ & - & 3041.94 & - \\
\hline & $v 2$ & $v(\mathrm{Cb}-\mathrm{Cb})$ & 1602 & 1602.04 & -0.04 \\
\hline & $v 3$ & $v(\mathrm{Ca}-\mathrm{Cm}) \mathrm{sym}$ & 1519 & 1525.06 & -6.06 \\
\hline & $v 4$ & $v$ (Pyrhalf-ring)sym & 1383 & 1383.45 & -0.45 \\
\hline & 15 & $v(\mathrm{Cb}-\mathrm{C}) \mathrm{sym}$ & 1025 & 1010.52 & 14.48 \\
\hline & 16 & $v$ (Pyr breathing) & 806 & 803.76 & 2.24 \\
\hline & $v 7$ & $\delta$ (Pyr def)sym & 674 & 685.99 & -11.99 \\
\hline & 18 & $v(\mathrm{Ni}-\mathrm{N})$ & 344 & 344.36 & -0.36 \\
\hline & 19 & $\delta(\mathrm{Cb}-\mathrm{C}) \mathrm{sym}$ & 226 & 225.66 & 0.34 \\
\hline \multirow{9}{*}{$\mathrm{B}_{1 \mathrm{~g}}$} & $v 10$ & $v^{\prime}(\mathrm{Ca}-\mathrm{Cm}) \mathrm{sym}$ & 1655 & 1639.26 & 15.74 \\
\hline & $v 11$ & $v(\mathrm{Cb}-\mathrm{Cb})$ & 1576 & 1577.96 & -1.96 \\
\hline & $v 12$ & $v$ (Pyr half-ring)sym & - & 1293.43 & - \\
\hline & $v 13$ & $\delta(\mathrm{Cm}-\mathrm{H})$ & 1220 & 1219.69 & 0.31 \\
\hline & $v 14$ & $v(\mathrm{Cb}-\mathrm{C}) \mathrm{sym}$ & - & 1065.55 & - \\
\hline & $v 15$ & $v$ (Pyr breathing) & - & 750.51 & - \\
\hline & $v 16$ & $\delta$ (Pyr def)sym & 751 & 752.43 & -1.43 \\
\hline & $v 17$ & $\delta(\mathrm{Cb}-\mathrm{C}) \mathrm{sym}$ & - & 304.08 & - \\
\hline & $v 18$ & $v(\mathrm{Ni}-\mathrm{N})$ & - & 423.15 & - \\
\hline \multirow{8}{*}{$\mathrm{A}_{2 \mathrm{~g}}$} & $v 19$ & $v^{\prime}(\mathrm{Ca}-\mathrm{Cm}) \mathrm{sym}$ & 1603 & 1589.26 & 13.74 \\
\hline & $v 20$ & $v$ (Pyr quarter-ring) & 1397 & 1396.89 & 0.11 \\
\hline & $v 21$ & $\delta(\mathrm{Cm}-\mathrm{H})$ & 1308 & 1327.40 & -19.40 \\
\hline & $v 22$ & $v^{\prime}($ Pyr half-ring)sym & 1121 & 1121.87 & -0.87 \\
\hline & $v 23$ & $v^{\prime}(\mathrm{Cb}-\mathrm{C})$ sym & - & 1104.44 & - \\
\hline & $v 24$ & $\delta^{\prime}($ Pyr def)sym & 739 & 732.81 & 6.19 \\
\hline & $v 25$ & $\delta$ (Pyr rot) & - & 523.70 & - \\
\hline & $v 26$ & $\delta^{\prime}(\mathrm{Cb}-\mathrm{C})$ sym & - & 382.51 & - \\
\hline \multirow{9}{*}{$\mathrm{B}_{2 \mathrm{~g}}$} & $v 27$ & $v(\mathrm{Cm}-\mathrm{H})$ & - & 3040.95 & - \\
\hline & $v 28$ & $v^{\prime}(\mathrm{Ca}-\mathrm{Cm}) \mathrm{sym}$ & - & 1507.32 & - \\
\hline & $v 29$ & $v$ (Pyr quarter-ring) & 1409 & 1408.53 & 0.47 \\
\hline & $v 30$ & $v^{\prime}$ (Pyr half-ring)sym & 1159 & 1142.34 & 16.66 \\
\hline & $v 31$ & $v^{\prime}(\mathrm{Cb}-\mathrm{C})$ sym & - & 1159.46 & - \\
\hline & $v 32$ & $\delta^{\prime}$ (Pyr def)sym & 785 & 773.06 & 11.94 \\
\hline & $v 33$ & $\delta$ (Pyr rot) & - & 528.26 & - \\
\hline & $v 34$ & $\delta^{\prime}(\mathrm{Cb}-\mathrm{C})$ sym & - & 437.96 & - \\
\hline & $v 35$ & $\delta($ Pyr transl $)$ & - & 178.96 & - \\
\hline \multirow{18}{*}{$\mathrm{Eu}$} & 136 & $v(\mathrm{Cm}-\mathrm{H})$ & - & 3040.95 & - \\
\hline & 137 & $v^{\prime}(\mathrm{Ca}-\mathrm{Cm}) \mathrm{sym}$ & 1604 & 1642.79 & -38.79 \\
\hline & $v 38$ & $v(\mathrm{Cb}-\mathrm{Cb})$ & 1557 & 1604.28 & -47.28 \\
\hline & 139 & $v(\mathrm{Ca}-\mathrm{Cm}) \mathrm{sym}$ & 1487 & 1474.88 & 12.12 \\
\hline & 140 & $v$ (Pyr quarter-ring) & 1443 & 1442.36 & 0.64 \\
\hline & $v 41$ & $v^{\prime}$ (Pyr half-ring)sym & 1389 & 1392.45 & -3.45 \\
\hline & $v 42$ & $\delta(\mathrm{Cm}-\mathrm{H})$ & 1268 & 1266.81 & 1.19 \\
\hline & $v 43$ & $v^{\prime}(\mathrm{Cb}-\mathrm{C})$ sym & 1148 & 1143.34 & 4.66 \\
\hline & 144 & $v^{\prime}$ (Pyr half-ring)sym & 1113 & 1113.39 & -0.39 \\
\hline & $v 45$ & $v^{\prime}(\mathrm{Cb}-\mathrm{C})$ sym & 993 & 994.40 & -1.60 \\
\hline & 146 & $\delta^{\prime}(\mathrm{Pyr}) \mathrm{sym}$ & 924 & 925.56 & -1.56 \\
\hline & 147 & $v$ (Pyr breathing) & 726 & 727.94 & -1.94 \\
\hline & 148 & $\delta$ (Pyr)sym & 605 & 604.92 & 0.08 \\
\hline & 149 & $\delta$ (Pyr rot) & 550 & 552.48 & -2.48 \\
\hline & 150 & $v(\mathrm{Ni}-\mathrm{N})$ & - & 501.94 & - \\
\hline & $\sqrt{51}$ & $\delta^{\prime}(\mathrm{Cb}-\mathrm{C})$ sym & - & 460.94 & - \\
\hline & $\sqrt{52}$ & $\delta(\mathrm{Cb}-\mathrm{C}) \mathrm{sym}$ & 287 & 288.26 & -1.26 \\
\hline & 153 & $\delta$ (Pyr transl) & - & 183.77 & - \\
\hline
\end{tabular}

${ }^{a}$ Experimental data has taken from Reference [24]. $\Delta$ (r.m.s) $=40.92 \mathrm{~cm}^{-1}$ 
Table 2. Comparison between the experimental and Calculated frequencies of the resonance Raman active fundamental modes of $\mathrm{Ni}(\mathrm{OEP})-\mathrm{d}_{4}\left(\mathrm{~cm}^{-1}\right)$.

\begin{tabular}{|c|c|c|c|c|c|}
\hline Symmetry & Mode & Description & $\operatorname{Exp}^{\mathrm{a}}$ & $\mathrm{Cal}$ & $\Delta($ Exp-Calc $)$ \\
\hline \multirow{9}{*}{$\mathrm{A}_{1 \mathrm{~g}}$} & $v 1$ & $v(\mathrm{Cm}-\mathrm{D})$ & - & 2265.10 & - \\
\hline & $v 2$ & $v(\mathrm{Cb}-\mathrm{Cb})$ & 1602 & 1612.99 & -10.99 \\
\hline & 13 & $v(\mathrm{Ca}-\mathrm{Cm}) \mathrm{sym}$ & 1512 & 1513.86 & -1.86 \\
\hline & 14 & $v$ (Pyr half-ring)sym & 1382 & 1384.58 & -2.58 \\
\hline & 15 & $v(\mathrm{Cb}-\mathrm{C})$ sym & 1026 & 1026.81 & -0.81 \\
\hline & 16 & $v$ (Pyr breathing) & 802 & 802.86 & -0.86 \\
\hline & 27 & $\delta$ (Pyr def)sym & 667 & 668.34 & -1.34 \\
\hline & 18 & $v(\mathrm{Ni}-\mathrm{N})$ & 342 & 340.76 & 1.24 \\
\hline & 19 & $\delta(\mathrm{Cb}-\mathrm{C}) \mathrm{sym}$ & 226 & 227.40 & -1.40 \\
\hline \multirow{9}{*}{$\mathrm{B}_{1 \mathrm{~g}}$} & $v 10$ & $v^{\prime}(\mathrm{Ca}-\mathrm{Cm}) \mathrm{sym}$ & 1645 & 1650.13 & -5.13 \\
\hline & $v 11$ & $v(\mathrm{Cb}-\mathrm{Cb})$ & 1576 & 1578.24 & -2.24 \\
\hline & $v 12$ & $v$ (Pyr half-ring)sym & - & 1272.24 & - \\
\hline & $v 13$ & $\delta(\mathrm{Cm}-\mathrm{D})$ & 950 & 951.44 & -1.44 \\
\hline & $v 14$ & $v(\mathrm{Cb}-\mathrm{C}) \mathrm{sym}$ & 1187 & 1188.94 & -1.94 \\
\hline & $v 15$ & $v$ (Pyr breathing) & - & 762.93 & - \\
\hline & $v 16$ & $\delta$ (Pyr def)sym & 684 & 683.18 & 0.82 \\
\hline & $v 17$ & $\delta(\mathrm{Cb}-\mathrm{C}) \mathrm{sym}$ & - & 296.58 & - \\
\hline & $v 18$ & $v(\mathrm{Ni}-\mathrm{N})$ & - & 171.41 & - \\
\hline \multirow{8}{*}{$\mathrm{A}_{2 \mathrm{~g}}$} & $v 19$ & $v^{\prime}(\mathrm{Ca}-\mathrm{Cm}) \mathrm{sym}$ & 1582 & 1565.75 & 16.25 \\
\hline & $v 20$ & $v$ (Pyr quater-ring) & 1397 & 1397.12 & -0.12 \\
\hline & $v 21$ & $\delta(\mathrm{Cm}-\mathrm{D})$ & 890 & 891.24 & -1.24 \\
\hline & $v 22$ & $v^{\prime}$ (Pyr half-ring)sym & 1202 & 1203.64 & -0.64 \\
\hline & $v 23$ & $v^{\prime}(\mathrm{Cb}-\mathrm{C}) \mathrm{sym}$ & 1029 & 1028.43 & 0.57 \\
\hline & $v 24$ & $\delta$ ' (Pyr def)sym & 733 & 736.96 & -3.96 \\
\hline & $v 25$ & $\delta$ (Pyr rot) & - & 524.78 & - \\
\hline & $v 26$ & $\delta^{\prime}(\mathrm{Cb}-\mathrm{C}) \mathrm{sym}$ & - & 277.35 & - \\
\hline \multirow{9}{*}{$\mathrm{B}_{2 \mathrm{~g}}$} & $v 27$ & $v(\mathrm{Cm}-\mathrm{D})$ & - & 2268.85 & - \\
\hline & $v 28$ & $v^{\prime}(\mathrm{Ca}-\mathrm{Cm}) \mathrm{sym}$ & - & 1712.19 & - \\
\hline & $v 29$ & $v($ Pyr quater-ring) & 1408 & 1408.90 & -0.90 \\
\hline & $v 30$ & $v^{\prime}$ (Pyr half-ring)sym & 1159 & 1159.91 & -0.91 \\
\hline & $v 31$ & $v^{\prime}(\mathrm{Cb}-\mathrm{C})$ sym & - & 1165.43 & - \\
\hline & $v 32$ & $\delta^{\prime}$ (Pyr def)sym & 785 & 805.59 & -20.59 \\
\hline & $v 33$ & $\delta$ (Pyr rot) & - & 493.73 & - \\
\hline & $v 34$ & $\delta^{\prime}(\mathrm{Cb}-\mathrm{C})$ sym & - & 252.37 & - \\
\hline & $v 35$ & $\delta($ Pyr transl) & - & 182.17 & - \\
\hline \multirow{18}{*}{$\mathrm{Eu}$} & 136 & $v(\mathrm{Cm}-\mathrm{D})$ & - & 3040.95 & - \\
\hline & 137 & $v^{\prime}(\mathrm{Ca}-\mathrm{Cm}) \mathrm{sym}$ & 1595 & 1592.94 & 2.06 \\
\hline & $v 38$ & $v(\mathrm{Cb}-\mathrm{Cb})$ & 1542 & 1543.50 & -1.50 \\
\hline & 139 & $v(\mathrm{Ca}-\mathrm{Cm})$ sym & 1480 & 1480.76 & -0.76 \\
\hline & $v 40$ & $v$ (Pyr quarter-ring) & 1440 & 1445.84 & -5.84 \\
\hline & $v 41$ & $v$ (Pyr half-ring)sym & 1383 & 1384.58 & -1.58 \\
\hline & $v 42$ & $\delta(\mathrm{Cm}-\mathrm{D})$ & 1175 & 1175.14 & -1.14 \\
\hline & $v 43$ & $v^{\prime}(\mathrm{Cb}-\mathrm{C}) \mathrm{sym}$ & 1114 & 1112.05 & 1.95 \\
\hline & $v 44$ & $v^{\prime}$ (Pyr half-ring)sym & 1018 & 1002.44 & 15.56 \\
\hline & $v 45$ & $v^{\prime}(\mathrm{Cb}-\mathrm{C}) \mathrm{sym}$ & 943 & 944.41 & -1.41 \\
\hline & $v 46$ & $\delta^{\prime}$ (Pyr)sym & 843 & 840.20 & 2.80 \\
\hline & $v 47$ & $v$ (Pyr breathing) & 722 & 723.01 & -0.01 \\
\hline & $v 48$ & $\delta$ (Pyr)sym & 597 & 598.28 & -1.28 \\
\hline & $v 49$ & $\delta$ (Pyr rot) & 537 & 536.54 & -1.95 \\
\hline & 150 & $v(\mathrm{Ni}-\mathrm{N})$ & - & 256.08 & - \\
\hline & $\sqrt{51}$ & $\delta^{\prime}(\mathrm{Cb}-\mathrm{C}) \mathrm{sym}$ & - & 302.32 & - \\
\hline & 152 & $\delta(\mathrm{Cb}-\mathrm{C}) \mathrm{sym}$ & - & 295.01 & - \\
\hline & 153 & $\delta(\mathrm{Pyr}$ transl) & - & 188.27 & - \\
\hline
\end{tabular}

${ }^{\mathrm{a}}$ Experimental data has taken from Reference [24]. $\Delta$ (r.m.s) $=33.03 \mathrm{~cm}^{-1}$ 
Table 3. Comparison between the experimental and Calculated frequencies of the resonance Raman active fundamental modes of $\mathrm{Ni}(\mathrm{OEP})-{ }^{15} \mathrm{~N}_{4}\left(\mathrm{~cm}^{-1}\right)$.

\begin{tabular}{|c|c|c|c|c|c|}
\hline Symmetry & Mode & Description & $\operatorname{Exp}^{a}$ & Cal & $\Delta($ Exp-Calc $)$ \\
\hline \multirow{9}{*}{$\mathrm{A}_{\lg }$} & $v 1$ & $v(\mathrm{Cm}-\mathrm{N})$ & - & 2089.8 & - \\
\hline & $v 2$ & $v(\mathrm{Cb}-\mathrm{Cb})$ & 1602 & 1603.02 & -1.02 \\
\hline & 13 & $v(\mathrm{Ca}-\mathrm{Cm}) \mathrm{sym}$ & 1519 & 1525.06 & -6.06 \\
\hline & $v 4$ & $v$ (Pyr half-ring)sym & 1377 & 1371.10 & 5.90 \\
\hline & 15 & $v(\mathrm{Cb}-\mathrm{C}) \mathrm{sym}$ & 1022 & 1021.12 & 0.88 \\
\hline & 16 & $v$ (Pyr breathing) & 801 & 803.76 & 2.76 \\
\hline & $v 7$ & $\delta$ (Pyr def)sym & 673 & 685.99 & -12.99 \\
\hline & 18 & $v(\mathrm{Ni}-\mathrm{N})$ & 344 & 344.26 & -0.26 \\
\hline & 19 & $\delta(\mathrm{Cb}-\mathrm{C})$ sym & 226 & 226.28 & -0.28 \\
\hline \multirow{9}{*}{$\mathrm{B}_{1 \mathrm{~g}}$} & $v 10$ & $v^{\prime}(\mathrm{Ca}-\mathrm{Cm}) \mathrm{sym}$ & 1655 & 1639.26 & 15.74 \\
\hline & $v 11$ & $v(\mathrm{Cb}-\mathrm{Cb})$ & 1576 & 1575.39 & 0.61 \\
\hline & $v 12$ & $v$ (Pyr half-ring)sym & - & 1337.81 & - \\
\hline & $v 13$ & $\delta(\mathrm{Cm}-\mathrm{N})$ & 1220 & 1220.05 & -0.05 \\
\hline & $v 14$ & $v(\mathrm{Cb}-\mathrm{C}) \mathrm{sym}$ & - & 1273.68 & - \\
\hline & $v 15$ & $v$ (Pyr breathing) & - & 750.51 & - \\
\hline & $v 16$ & $\delta$ (Pyr def)sym & 749 & 752.43 & -3.43 \\
\hline & $v 17$ & $\delta(\mathrm{Cb}-\mathrm{C})$ sym & - & 330.59 & - \\
\hline & $v 18$ & $v(\mathrm{Ni}-\mathrm{N})$ & - & 369.38 & - \\
\hline \multirow{8}{*}{$\mathrm{A}_{2 \mathrm{~g}}$} & $v 19$ & $v^{\prime}(\mathrm{Ca}-\mathrm{Cm}) \mathrm{sym}$ & 1603 & 1589.26 & 13.74 \\
\hline & $v 20$ & $v$ (Pyr quater-ring) & 1396 & 1396.89 & -0.89 \\
\hline & $v 21$ & $\delta(\mathrm{Cm}-\mathrm{N})$ & 1305 & 1309.28 & -4.28 \\
\hline & $v 22$ & $v^{\prime}$ (Pyr half-ring)sym & 1108 & 1113.39 & -5.39 \\
\hline & $v 23$ & $v^{\prime}(\mathrm{Cb}-\mathrm{C})$ sym & - & 1065.55 & \\
\hline & $v 24$ & $\delta^{\prime}$ (Pyr def)sym & 732 & 732.81 & -0.81 \\
\hline & $v 25$ & $\delta$ (Pyr rot) & - & 523.70 & - \\
\hline & $v 26$ & $\delta^{\prime}(\mathrm{Cb}-\mathrm{C})$ sym & - & 304.08 & - \\
\hline \multirow{9}{*}{$\mathrm{B}_{2 \mathrm{~g}}$} & $v 27$ & $v(\mathrm{Cm}-\mathrm{N})$ & - & 2105.12 & - \\
\hline & $v 28$ & $v^{\prime}(\mathrm{Ca}-\mathrm{Cm}) \mathrm{sym}$ & - & 1474.88 & - \\
\hline & $v 29$ & $v$ (Pyr quater-ring) & 1408 & 1408.53 & -0.53 \\
\hline & v30 & $v^{\prime}$ (Pyr half-ring)sym & 1150 & 1142.34 & 7.66 \\
\hline & $v 31$ & $v^{\prime}(\mathrm{Cb}-\mathrm{C}) \mathrm{sym}$ & - & 1010.52 & - \\
\hline & $v 32$ & $\delta^{\prime}$ (Pyr def)sym & 785 & 773.06 & 11.94 \\
\hline & $v 33$ & $\delta$ (Pyr rot) & - & 528.26 & - \\
\hline & $v 34$ & $\delta^{\prime}(\mathrm{Cb}-\mathrm{C}) \mathrm{sym}$ & - & 460.94 & - \\
\hline & v35 & $\delta($ Pyr transl $)$ & - & 178.96 & - \\
\hline \multirow{18}{*}{$\mathrm{Eu}$} & $v 36$ & $v(\mathrm{Cm}-\mathrm{N})$ & - & 2120.38 & - \\
\hline & $v 37$ & $v^{\prime}(\mathrm{Ca}-\mathrm{Cm}) \mathrm{sym}$ & 1603 & 1603.02 & -0.02 \\
\hline & v38 & $v(\mathrm{Cb}-\mathrm{Cb})$ & 1555 & 1562.23 & -7.23 \\
\hline & $v 39$ & $v(\mathrm{Ca}-\mathrm{Cm}) \mathrm{sym}$ & 1484 & 1483.30 & 0.70 \\
\hline & $v 40$ & $v$ (Pyr quarter-ring) & 1442 & 1442.36 & 0.36 \\
\hline & $v 41$ & $v$ (Pyr half-ring)sym & 1386 & 1383.45 & 2.55 \\
\hline & $v 42$ & $\delta(\mathrm{Cm}-\mathrm{N})$ & 1266 & 1265.11 & 0.89 \\
\hline & $v 43$ & $v^{\prime}(\mathrm{Cb}-\mathrm{C}) \mathrm{sym}$ & 1140 & 1147.40 & -7.40 \\
\hline & $v 44$ & $v^{\prime}$ (Pyr half-ring)sym & 1108 & 1113.39 & -5.39 \\
\hline & 145 & $v^{\prime}(\mathrm{Cb}-\mathrm{C}) \mathrm{sym}$ & 986 & 994.40 & -8.40 \\
\hline & 146 & $\delta^{\prime}$ (Pyr)sym & 921 & 918.06 & 2.94 \\
\hline & 147 & $v$ (Pyr breathing) & 719 & 727.94 & -8.94 \\
\hline & 148 & $\delta$ (Pyr)sym & 602 & 601.10 & 0.90 \\
\hline & 149 & $\delta$ (Pyr rot) & 550 & 552.48 & -2.48 \\
\hline & 150 & $v(\mathrm{Ni}-\mathrm{N})$ & - & 394.50 & - \\
\hline & 151 & $\delta^{\prime}(\mathrm{Cb}-\mathrm{C}) \mathrm{sym}$ & - & 374.90 & - \\
\hline & 152 & $\delta(\mathrm{Cb}-\mathrm{C}) \mathrm{sym}$ & - & 288.26 & - \\
\hline & 153 & $\delta($ Pyr transl $)$ & - & 183.77 & - \\
\hline
\end{tabular}

${ }^{a}$ Experimental data has taken from Reference [24]. $\Delta$ (r.m.s) $=4.04 \mathrm{~cm}^{-1}$. 
Table 4. Comparisons between the experimental and calculated frequencies of the Raman active fundamental modes of $\mathrm{C}_{60}$ $\left(\mathrm{cm}^{-1}\right)$.

\begin{tabular}{|c|c|c|c|}
\hline Vibrational mode & $\operatorname{Exp}^{b}$ & $\mathrm{Cal}$ & $\Delta($ Exp-Calc $)$ \\
\hline$v 1$ & 273 & 275.9303 & -2.9303 \\
\hline$v 2$ & 497 & 498.3048 & -1.3048 \\
\hline 13 & 528 & 530.2039 & -2.2039 \\
\hline 14 & 577 & 577.3049 & -0.3049 \\
\hline 15 & 1183 & 1182.2093 & 0.7907 \\
\hline 16 & 1429 & 1431.9848 & -2.9848 \\
\hline 27 & 1469 & 1470.5968 & -1.5968 \\
\hline
\end{tabular}

$\Delta$ (r.m.s) $=6.439 \mathrm{~cm}^{-1}$.

Table 5. Comparisons between the experimental and Calculated frequencies of the Raman active fundamental modes of $\mathrm{C}_{70}$ $\left(\mathrm{cm}^{-1}\right)$.

\begin{tabular}{cccc}
\hline Vibrational mode & Exp $^{\mathrm{b}}$ & Cal & $\Delta($ Exp-Calc) \\
\hline$v 1$ & 260 & 259.3543 & 0.6457 \\
$v 2$ & 571 & 573.0294 & -2.0294 \\
$v 3$ & 1062 & 1064.3029 & -2.3029 \\
$v 4$ & 1185 & 1186.0928 & -1.0928 \\
$v 5$ & 1232 & 1233.2930 & -1.2930 \\
$v 6$ & 1513 & 1513.2087 & -2.9848 \\
$v 7$ & 1568 & 1565.3392 & 2.6608 \\
\hline
\end{tabular}

${ }^{\mathrm{b}}$ Experimental data has taken from Reference[25], $\Delta$ (r.m.s) $=3.2029 \mathrm{~cm}^{-1}$.

Table 6. Fitting algebraic parameters of octaethylporphyrinato Ni(II) and its meso-deuterated and N-substituted derivatives.

\begin{tabular}{|c|c|c|c|c|c|c|c|c|c|c|}
\hline & $\mathrm{Cm}-\mathrm{H}$ & $\mathrm{Cb}-\mathrm{Cb}$ & $\mathrm{Cb}-\mathrm{C}$ & $\mathrm{Ca}-\mathrm{Cm}$ & Ni-N & Pyr.half & Pyr.quater & Pyr.breath & Pyr.rot & Pyr.def \\
\hline \multicolumn{11}{|c|}{$\mathrm{Ni}(\mathrm{OEP})$ molecule } \\
\hline A & -1.8972 & -1.7829 & -1.8293 & -1.5403 & -2.2832 & -1.0293 & -2.3940 & -1.2930 & -1.2394 & -1.2930 \\
\hline$A^{\prime}$ & -0.3094 & -0.3049 & -0.3833 & -0.3209 & -0.4954 & -0.4859 & -0.4930 & -0.4938 & -0.2918 & -0.3820 \\
\hline$\lambda$ & 0.0394 & 0.0238 & 0.0495 & 0.0594 & 0.0293 & 0.0433 & 0.0867 & 0.0594 & 0.0637 & 0.0322 \\
\hline$\lambda$ & 0.1029 & 0.0384 & 0.3902 & 0.0293 & 0.0390 & 0.0902 & 0.0293 & 0.0783 & 0.0394 & 0.9200 \\
\hline \multicolumn{11}{|c|}{$\mathrm{Ni}(\mathrm{OEP})-\mathrm{d}_{4}$ molecule } \\
\hline $\mathrm{A}$ & -1.9567 & -1.7394 & -1.7574 & -1.4839 & -2.4758 & -1.9438 & -1.5783 & -1.4839 & -1.3489 & -1.4938 \\
\hline$A^{\prime}$ & -0.4039 & -0.5493 & -0.4938 & -0.2345 & -0.5489 & -0.2390 & -0.4465 & -0.3493 & -0.2930 & -0.4930 \\
\hline$\lambda$ & 0.0840 & 0.0349 & 0.0657 & 0.0405 & 0.0349 & 0.0128 & 0.0928 & 0.0647 & 0.0493 & 0.0574 \\
\hline$\lambda^{\prime}$ & 0.2349 & 0.0504 & 0.0394 & 0.0192 & 0.0128 & 0.0495 & 0.0112 & 0.0349 & 0.0325 & 0.0932 \\
\hline \multicolumn{11}{|c|}{$\mathrm{Ni}(\mathrm{OEP})-15 \mathrm{~N}_{4}$ molecule } \\
\hline $\mathrm{A}$ & -1.7849 & -1.7839 & -1.8495 & -1.3849 & -2.3948 & -1.0490 & -2.4930 & -1.3049 & -1.3829 & -1.2389 \\
\hline$A^{\prime}$ & -0.4302 & -0.3940 & -0.3647 & -0.2784 & -0.4304 & -0.3920 & -0.4289 & -0.3940 & -0.3920 & -0.4673 \\
\hline$\lambda$ & 0.0333 & 0.0394 & 0.0432 & 0.0394 & 0.0239 & 0.0320 & 0.0788 & 0.0403 & 0.0433 & 0.0333 \\
\hline$\lambda^{\prime}$ & 0.0938 & 0.0574 & 0.2987 & 0.0293 & 0.0293 & 0.0843 & 0.0392 & 0.0563 & 0.0233 & 0.0945 \\
\hline
\end{tabular}

Table 7. Fitting algebraic parameters of fullerenes $\mathrm{C}_{60}$ and $\mathrm{C}_{70}$.

\begin{tabular}{ccccc}
\hline & Vibron number & & \multicolumn{2}{c}{ Algebraic parameters } \\
\hline & $\mathrm{N}$ & $\mathrm{A}$ & $\mathrm{A}^{\prime}$ & $\lambda$ \\
$\mathrm{C} 60$ & 140 & -1.4309 & 0.0384 & 0.0739 \\
$\mathrm{C} 70$ & 140 & 0.9837 & 0.0456 & 0.0348 \\
\hline
\end{tabular}

All values in $\mathrm{cm}^{-1}$ except $\mathrm{N}$, which is dimensionless. 


\section{ACKNOWLEDGEMENTS}

The author Srinivasa Rao Karumuri would like to thank Prof. Thomson G. Spiro for providing necessary literature for this study.

The author Srinivasa Rao Karumuri also would like to thank University of Grant Commission (UGC), New Delhi, India, for providing the financial assistance for this study.

\section{REFERENCES}

[1] Kroto, H.W., Heath, J.R., Brien, S.C.O., Curl, R.F. and Smalley, R.E. (1985) $\mathrm{C}_{60}$ : Buckminsterfullerene. Nature, 318, 162-163. doi:10.1038/318162a 0

[2] Treubig Jr., J.M. and Brown, P.R. (2002) Analysis of $\mathrm{C}_{60}$ and $\mathrm{C}_{70}$ fullerenes using high-performance liquid chromatography-Fourier transform infrared spectroscopy. Journal of Chromatography A, 960, 135-142.

[3] Levine, R.D. (1982) Representation of one-dimensional motion in a morse potential by a quadratic Hamiltonian. Chemical Physics Letters, 95, 87-90. doi:10.1016/0009-2614(83)85071-4

[4] Iachello, F. and Levine, R.D. (1982) Algebraic approach to molecular rotation-vibration spectra. I. Diatomic molecules. Journal of Chemical Physics, 77, 3046-3055. doi:10.1063/1.444228

[5] van Roosmalen, O.S., Dieperink, A.E.L. and Iachello, F. (1982) A unified algebraic model description for interacting vibrational modes in ABA molecules. Chemical Physics Letters, 85, 32-36. doi:10.1016/0009-2614(82)83455-6

[6] van Roosmalen, O.S., Iachello, F., Levine, R.D. and Dieperink, A.E.L. (1983) The geometrical-classical limit of algebraic Hamiltonians of molecular vibrational spectra. Chemical Physics Letters, 79, 2515. doi:10.1063/1.446164

[7] Sarkar, N.K., Choudhury, J. and Bhattacharjee, R. (2006) An algebraic approach to the study of the vibrational spectra of HCN. Molecular Physics, 104, 3051-3055. doi:10.1080/00268970600954235

[8] Sarkar, N.K., Choudhury, J., Karumuri, S.R. and Bhattacharjee, R. (2008) An algebraic approach to the comparative study of the vibrational spectra of monofluoroacetylene (HCCF) and deuterated acetylene (HCCD). Molecular Physics, 106, 693-702. doi:10.1080/00268970801939019

[9] Sarkar, N.K., Choudhury, J. and Bhattacharjee, R. (2008) Study of vibrational spectra of some linear triatomic molecules. Indian Journal of Physics, 82, 767.

[10] Choudhury, J., Karumuri, S.R., Sarkar, N.K. and Bhattacharjee, R. (2008) Vibrational spectroscopy of $\mathrm{CCl}_{4}$ and $\mathrm{SnBr}_{4}$ using lie algebraic approach. Physics and $\mathrm{As}$ tronomy, 71, 439-445. doi:10.1007/s12043-008-0123-Z

[11] Choudhury, J., Karumuri, S.R. and Bhattacharjee, R., (2008) Algebraic approach to analyze the vibrational spectra of tetrahedral molecules. Indian Journal of Physics, 82, 561-565.

[12] Karumuri, S.R., Sarkar, N.K., Choudhury, J. and Bhat- tacharjee, R. (2008) Vibrational spectroscopy of $\mathrm{C}_{\mathrm{m}}-\mathrm{H}$, $\mathrm{C}_{\beta} \mathrm{C}_{\beta}$ stretching vibrations of Nickel metalloporphyrins. Molecular Physics, 106, 1733-1738. doi: $10.1080 / 00268970802248998$

[13] Karumuri, S.R., Choudhury, J., Sarkar, N.K. and Bhattacharjee, R. (2008) Analysis of resonance raman spectra of nickeloctaethyl porphyrin using lie algebra. Journal of Environmental Research and Development, 3, 250-256.

[14] Karumuri, S.R., Sarkar, N.K., Choudhury, J. and Bhattacharjee, R. (2009) Study of vibrational spectra of Nickel metalloporphyrins: An algebraic approach. PramanaJournal of Physics, 72, 517-525.

[15] Karumuri, S.R., Sarkar, N.K., Choudhury, J. and Bhattacharjee, R. (2009) Vibrational spectroscopy of stretching and bending modes of nickel tetraphenyl porphyrin: An algebraic approach. Chinese Physics Letters, 26, 093301. doi:10.1088/0256-307X/26/9/093301

[16] Karumuri, S.R., Sarkar, N.K., Choudhury, J. and Bhattacharjee, R. (2009) U(2) algebraic model applied to vibrational spectra of Nickel Metalloporphyrins. Journal of Molecular Spectroscopy, 255, 183-188. doi:10.1016/j.jms.2009.03.014

[17] Karumuri, S.R., Choudhury, J., Sarkar, N.K. and Bhattacharjee, R. (2010) Vibrational Spectroscopy of $\mathrm{C}_{\mathrm{m}}-\mathrm{C} /$ $\mathrm{C}_{\mathrm{b}}-\mathrm{C}_{\mathrm{b}}$ stretching vibrations of Copper Tetramesityl Porphyrin $\mathrm{Cu}$ (TMP): An algebraic approach. Pramana-Journal of Physics, 74, 57-66. doi:10.1007/s12043-010-0007-x

[18] Karumuri, S.R. (2010) Calculation of vibrational spectra by an algebraic approach: Applications to Copper Tetramesityl Porphyrins and its Cation radicals. Journal of Molecular Spectroscopy, 259, 86-92. doi:10.1016/i.jms.2009.11.005

[19] Iachello, F. and Levine, R.D. (1995) Algebraic theory of molecules. Oxford University Press, Oxford.

[20] Iachello, F. and Oss, S. (2002), Algebraic methods in quantum mechanics: From molecules to polymers. Physics and Astronomy, 19, 307-314. doi:10.1140/epjd/e20020089

[21] Child, M.S. and Halonen, L.O. (1984) Overtone frequencies and intensities in the local mode picture. Advances in Chemical Physics, 57, 1. doi:10.1002/9780470142813.ch1

[22] Wood, B.R., Stoddart, P.R. and McNaughton, D. (2007) Molecular imaging of red blood cells by raman spectroscopy. Australian Journal of Chemistry, 387, 1691

[23] Phuber, K. and Herzberg, G., (1979) Molecular spectra and molecular structure IV: Constants of diatomic molecules. Van Nostrand Reinhold Co., New York.

[24] Kitagawa, T., Abe, M. and Ogoshi, H. (1978) Resonance Raman spectra of octaethylporphyrinato- $\mathrm{Ni}$ (II) and mesodeuterated and ${ }^{15} \mathrm{~N}$ substituted derivatives. II. A normal coordinate analysis. Journal of Chemical Physics, 69, 4526. doi:10.1063/1.436450

[25] Schettino, V., Pagliai M. and Cardini, G. (2002) The infrared and raman spectra of fullerene $\mathrm{C}_{70}$. DFT calculations and correlation with $\mathrm{C}_{60}$. The Journal of Physical Chemistry A, 106, 1815-1823. doi:10.1021/jp012680d. 